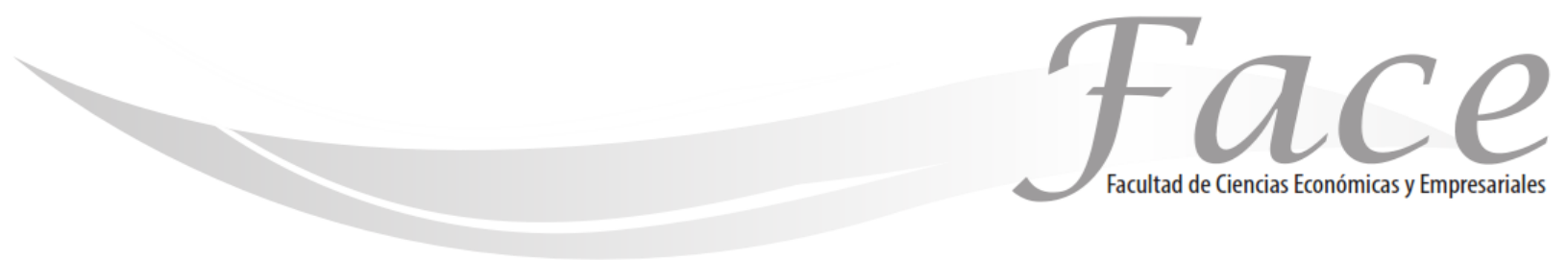

ISSN Impreso: 1794-9920 ISSN Electrónico: 2500-9338

Volumen $18-\mathrm{N}^{\circ} 2$

Año 2018

Págs. 39 - 49

\title{
EMPRENDEDURISMO DESDE LA PERSPECTIVA DE DOS GRUPOS DE UNIVERSITARIOS: MÉXICO VS ECUADOR *
}

\author{
Marco Francisco Martínez Aguilar ** \\ Enlace ORCID: https://orcid.org/0000-0002-3993-2513 \\ Ledys Hernández Chacón *** \\ Enlace ORCID: https:// orcid.org/0000-0002-3706-1873 \\ Mónica Eugenia Peñalosa Otero **** \\ Enlace ORCID: http://orcid.org/0000-0002-2208-9224
}

Fecha de Recepción: 22 de Septiembre 2018

Fecha de Aprobación: 27 de Diciembre 2018

\section{Resumen:}

Investigación resultante de los convenios de investigación entre la Universidad Autónoma de San Luis Potosí campus Altiplano y la Universidad de Otavalo Ecuador. Se trata de una investigación cuantitativa descriptiva y comparada entre grupos que detecta la percepción de factores individuales y del entorno cercano relacionado a las expectativas de emprendimiento futuro de negocios. Encuentra que servicios, alimentos y comercio son los negocios para emprender, informa de las necesidades de capacitación de los futuros emprendedores y describe las motivaciones a emprender en primer lugar la superación personal, en segundo la familia y en tercero el dinero.

Palabras clave: Emprendimiento, expectativas, capacitación, motivación

\footnotetext{
* Artículo de reflexión resultado de la investigación que busca detectar y comparar la percepción que se tiene de factores individuales y del entorno cercano relacionado a las expectativas de emprendimiento futuro de negocios en México y Ecuador.

** Maestro en educación adscrito a la Coordinación Académica Región Altiplano. Universidad Autónoma de San Luis Potosí. México. Correo electrónico: marcos.martinez@uaslp.mx

**** Psicóloga y Profesora de la Universidad de Otavalo, Carrera de Desarrollo Social y Cultural, Campus Cdla Imbaya, Ecuador. Correo electrónico: Ihernandez@uotavalo.edu.ec

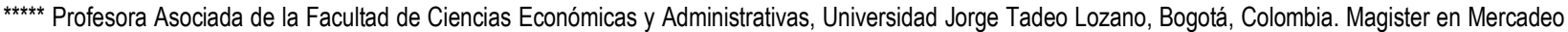
Agroindustrial. Profesional en Relaciones Internacionales. Investigadora Jr. de Colciencias. Se ha venido desempeñando en los últimos años como Docente Investigadora en la Universidad Jorge Tadeo Lozano, la Universidad de La Salle y la Universidad de Pamplona - Colombia. Correo electrónico: monica.penalosa@utadeo.edu.co
} 


\title{
ENTREPRENEURSHIP FROM THE PERSPECTIVE OF TWO UNIVERSITY GROUPS: MEXICO VS ECUADOR
}

\begin{abstract}
:
Research resulting from the research agreements between the Autonomous University of San Luis Potosí Altiplano campus and the University of Otavalo Ecuador. It is a descriptive and comparative quantitative research between groups that detects the perception of individual factors and the near environment related to the expectations of future business entrepreneurship. Finds what services, food and trade are the businesses to be undertaken, informs about the training needs of future entrepreneurs and describes the motivations to undertake first personal improvement, second the family and third economic gain.
\end{abstract}

Keywords: Entrepreneurship, expectations, training, motivation.

\section{EMPREENDEDORISMO NA PERSPECTIVA DE DOIS GRUPOS UNIVERSITÁRIOS: MÉXICO CONTRA EQUADOR}

\section{Resumo:}

Pesquisa resultante dos acordos de pesquisa entre o campus Altiplano da Universidade Autônoma de San Luis Potosí e a Universidade de Otavalo, Equador. Trata-se de uma pesquisa quantitativa descritiva e comparativa entre grupos que detecta a percepção de fatores individuais e o ambiente próximo relacionado às expectativas de empreendedorismo empresarial futuro. Encontra quais serviços, alimentos e comércio são os negócios a serem realizados, informa sobre as necessidades de treinamento de futuros empreendedores e descreve as motivações para empreender a primeira melhoria pessoal, segundo a família e o terceiro dinheiro.

Palavras-chave: Empreendedorismo, expectativas, treinamento, motivação. 


\section{INTRODUCCIÓN:}

Las sociedades en vías de desarrollo se han ido incrementando, hasta hace no más de 20 años muchos países catalogados como limitados en cuanto a recursos y educación apostaban a la industrialización como método para salir de los estados de limitación económica ante los países desarrollados. Los eventos económicos y sociales en diversas áreas del mundo como el desastre de las torres gemelas, la caída de las tasas de interés, el cambio del sistema económico en china, las guerras del petróleo y la caída de los precios del energético dejaron a estas sociedades "en desarrollo" nuevamente ante el dilema de cómo mejorar los estados de limitación de sus sociedades.

La nueva apuesta es la capacitación y el desarrollo de lo que se ha llamado emprendimiento, emprendedurismo 0 microempresario. Diversos países y sociedades han tratado de estimular esta área de la economía tratando de destrabar el problema económico a través de la capacitación de personas que en muchos casos luego de ser despedidos y por diversos motivos (edad, preparación, expectativa económica) no se han podido colocar en un nuevo empleo, también se ha tratado de fortalecer a egresados de niveles técnicos y superiores para que a partir de sus conocimientos generen sus propias fuentes de trabajo. La inversión en diversos países ha sido amplia, recursos de capacitación en muchos casos sin costo, apoyos a fondo perdido y las llamadas incubadoras han tratado de fortalecer la actividad del emprendimiento; en pero muchos de los negocios no llegan a sostenerse y evolucionar.

El presente trabajo intenta hacer una descripción de lo que dos grupos de Universitarios piensan de ser emprendedor. El análisis se centra en tres áreas principales, la primera que toma los aspectos que aquí se denominan individuales personales, el segundo perspectiva del entorno cercano (amigos, familia, sociedad en la que se mueve) y el tercero referente al análisis de la expectativa social de la sociedad en que habita (este último no analizado en el presente trabajo).

Los participantes son estudiantes universitarios de dos campus uno en San Luis Potosí México y otro en Imbabura Ecuador, aplicado en ciudades de 100000 habitantes aproximadamente con características ambas de estar inmersos en una población donde el comercio es la principal actividad de la región.

\section{MARCO TEÓRICO:}

Uno de los primeros documentos que llama la atención hacia el emprendimiento es el del autor Cantillon en 1755 donde en su apartado XIII describe la nueva actividad del empresario o emprendedor "Cela fait que plusieurs personnes dans la ville s'érigent en marchands ou entrepreneurs, pour acheter les denrées de la campagne de ceux qui les apportent, ou pour les faire apporter pour leur compte" (Cantillon. 2011). "Esto hace que muchas personas en la ciudad se conviertan en vendedores 0 empresarios, para comprar la comida del campo de aquellos que la traen, o para que la traigan en su nombre".

Ya en aquella lejana época la posibilidad del comercio o el desarrollo de actividades empresariales o emprendedoras se enfocaba a la intermediación. A lo largo de la historia los modelos de producción han reorientado los estilos de empresa y las sociedades han cruzado por los modelos artesanal, industrial, post industrial y el de la era de la globalización.

Si bien todos han permitido desarrollos en su momento es claro que en todos siempre ha habido un cumulo de comerciantes, productores, transformadores que buscan ya sea por motivos sociales 0 individuales la independencia. Estos son los llamados emprendedores. Para Kirzner la actividad de empresarios y emprendedores no solo está marcada por los eventos económicos de las sociedades y dice "toda acción humana está presente un elemento que, aunque es crucial para la actividad economizante en general, no se puede analizar en términos de economía, maximización o con criterios de eficiencia" (Huerta 1986).

Existen sinnúmero de definiciones de emprendimiento, Torralba en su trabajo factores clave en el desarrollo del emprendimiento lo define "La búsqueda, evaluación y explotación de oportunidades en el mercado a partir de la innovación, bajo unas circunstancias de futuro incierto y con una gran probabilidad de fracaso. El objetivo final será obtener un beneficio extraordinario ya sea económico o social" (2014). Para el mismo autor "la formación no resulta un factor clave para el emprendimiento en sí, pero es el medio a través del cual conseguir una cultura que favorezca el emprendimiento" (Torralba. 2014) 
Entre los factores psicológicos que se pueden contemplar son la autoimagen, seguridad y los antecedentes individuales del emprendedor 0 futuro emprendedor. McClelland sostenía que los tres factores individuales en los individuos son las necesidades de logro, poder y afiliación y que ellas son el factor detonante en la motivación hacia conductas dirigidas. En su libro de comportamiento organizacional Amorós basado en la teoría de Gardner afirma que los sujetos motivados "Buscan situaciones, en las que tengan la responsabilidad personal de brindar soluciones a los problemas, situaciones en las que pueden recibir una retroalimentación rápida acerca de su desempeño, a fin de saber si están mejorando, situaciones con metas desafiantes" (Amorós, 2007). Parra. Rubio y López (2017) refieren que los estudios sobre emprendimiento han contado con al menos veintiocho orientaciones de estudio y autores sobre los factores que los emprendedores presentan o no.

Desde la propuesta de este equipo los factores del microentorno son los que impactan de manera inmediata en la perspectiva e interés por convertirse en emprendedores en los sujetos, El microentorno desde el punto de vista de Kotler y Armstrong (2008) "Consiste en fuerzas cercanas a la empresa compañía / individuo, proveedores, intermediarios de marketing, mercados de clientes, competidores y públicos que inciden en su capacidad de servir al cliente", esta será la primera influencia percibida, en tanto que los factores más lejanos o menos percibidos por los emprendedores no serán tomados en cuenta en la decisión de emprendimiento.

Por otro lado, probablemente los prospectos de emprendedor no demeritan los factores más globales, sin embargo, no les toman en cuenta para determinar su decisión futura de emprender como prioritaria. Los mismos Kotler \& Armstrong definen macro entorno así "Grandes fuerzas de la sociedad demográficas, económicas, naturales, tecnológicas, políticas y culturales que afectan al microentorno".

\section{METODOLOGÍA:}

Se trata de un diseño transeccional descriptivo pues "indaga las incidencias de modalidades o niveles de una 0 más variables en una población" (Hernández, Fernández y Baptista 2010). Además, relaciona a dos poblaciones de características similares para encontrar concordancias 0 divergencias entre ambas. La selección de la muestra fue dirigida a dos poblaciones de estudiantes de nivel académico de licenciatura en estudio.

Otro elemento característico de ambas poblaciones es la densidad poblacional de las que las muestras provienen, encontrándose para ambos casos una demografía de aproximadamente 100, 000 habitantes dentro de las ciudades de Otavalo Ecuador y Matehuala México. La selección de participantes fue por invitación general a las poblaciones estudiantiles y las muestras fueron analizadas como un $100 \%$ equilibrando equitativamente las muestras por género para ambas poblaciones participantes. Se contó con un total de 532 entrevistas lo que arroja un C=95\% y $e=4.3$. mediante el cálculo de muestra para poblaciones infinitas con $p$ e $q=.5$

La muestra fue recolectada en procedimiento cara a cara entre los meses de junio y julio de 2018 dentro de las instalaciones de la U. de Otavalo Ecuador y la Universidad Autónoma de San Luis Potosí, campus COARA. México. El proyecto se desarrolló mediante trabajo de convenio entre ambas instituciones en el desarrollo de estancia académica.

Este trabajo analiza los doce primeros factores del agrupamiento de treinta y cuatro que componen el total de variables del proyecto agrupadas en factores individuales, entorno inmediato y entorno regional. Los factores se describen en la siguiente tabla. 
Tabla 1. Factores del emprendedurismo potencial COARA /

Otavalo

\begin{tabular}{|c|c|c|}
\hline Elementos individuales & Entorno inmediato & Entorno regional \\
\hline $\begin{array}{l}\text { Antecedentes de compra venta, } \\
\text { innovación percibida, tendencia a } \\
\text { ahorro, espíritu de aventura, } \\
\text { entusiasmo por actividad, } \\
\text { expectativa de emprendimiento, } \\
\text { positivismo, riesgo, carácter y } \\
\text { personalidad. }\end{array}$ & $\begin{array}{l}\text { Influencias familiares, estilos de gasto/ahorro, } \\
\text { presencia de emprendedores cercanos, } \\
\text { oportunidad en el entorno, autopercepción de } \\
\text { habilidad de negocios, respaldo económico } \\
\text { familiar, cuento con capacitación necesaria, } \\
\text { suerte, facilidades entorno cercano, capacidad } \\
\text { de planeación y espera. }\end{array}$ & $\begin{array}{l}\text { Ambiente prometedor, tendencias laborales del } \\
\text { medio, independencia laboral, percepción de } \\
\text { burocracia y gobierno, apoyos gubernamentales, } \\
\text { estatus social de emprendedores, estatus social } \\
\text { de emprendedores, medios de comunicación } \\
\text { fortaleciendo emprendimiento. No analizado en } \\
\text { este trabajo }\end{array}$ \\
\hline
\end{tabular}

\section{RESULTADOS Y DISCUSIÓN:}

A continuación, se detallan los resultados por cada una de las áreas del trabajo. El análisis describe los totales por país y su división de resultado por género, dentro del mismo se efectúa algunas puntualizaciones por tema.

En el área individual se contemplaron 10 aspectos que se describen a continuación de manera independiente y comparada para ambos grupos.

\section{ELEMENTOS INDIVIDUALES}

Se cuestionó a los entrevistados ¿Antes de los 10 años compraste $y$ vendiste algo para darte un gusto o por alguna necesidad? Los resultados generales son $43.1 \%$ de los participantes mexicanos y $56.5 \%$ de los ecuatorianos lo hicieron. De estas cifras el análisis por género nos indica que el $47.1 \%$ de los hombres Mexicanos y el $60.9 \%$ de los ecuatorianos y el $39.1 \%$ de las Mexicanas y el $52.2 \%$ de las ecuatorianas compraron 0 vender algún producto para satisfacer una necesidad 0 deseo.

\begin{abstract}
¿Juntaste dinero antes de los 10 años para comprar y revender algo? Aquí la cifra arroja que el $39.7 \%$ de los mexicanos y el $54.3 \%$ de los ecuatorianos ahorro en etapas tempranas con la intensión de negociar. Por género $42 \%$ de los hombres mexicanos y $59.8 \%$ de los ecuatorianos y $37.4 \%$ de las mexicanas y $48.9 \%$ de las ecuatorianas contestaron de manera positiva este reactivo.
\end{abstract}

Para acentuar en el tema anterior se usó el reactivo siguiente: Regularmente trato de ahorrar. Las respuestas son que el $80.5 \%$ de los mexicanos y $75 \%$ de los Ecuatorianos al menos tienen la intensión de ahorro, por genero $78.7 \%$ de los hombres mexicanos y $77.2 \%$ de los ecuatorianos y $82.2 \%$ de las mexicanas y $72.8 \%$ de las ecuatorianas intentaron ahorrar.

\section{Carácter y personalidad}

Los rasgos de carácter intervienen de manera importante en la continuidad de los negocios que surgen todos los días, el carácter positivo, la motivación, la disposición de personalidad es fundamental. Se cuestionó en esta área con los siguientes aspectos.

Los que me conocen dicen que soy entusiasta El 68.1\% de los respondientes mexicanos y el $73.4 \%$ de los ecuatorianos afirmaron serlo, por género $63.8 \%$ de los hombres mexicanos y $76.1 \%$ de los ecuatorianos y $72.4 \%$ de las mexicanas y $70.7 \%$ de las ecuatorianas afirman en el mismo sentido. 
Ante la afirmación soy aventurero $73.9 \%$ de los mexicanos y $72.3 \%$ de los ecuatorianos responden de manera positiva. Por país los hombres mexicanos serlo en el $71.8 \%$ de los casos y entre los ecuatorianos el $77.2 \%$. entre las mujeres y en el mismo orden las respuestas positivas fueron $75.9 \%$ y $67.4 \%$.

La relación de consumo está ligada a la personalidad definitivamente, en los cuestionarios siguientes se ratifica. El carácter emprendedor en muchos casos requiere no ser el primero, sino un análisis que permita asumir riesgos calculados.

Me gusta ser innovador y trato de comprar lo más nuevo en el mercado; Del grupo de participantes mexicanos $37.9 \%$ y del ecuatoriano $51.6 \%$ afirmaron tratar de comprar lo más nuevo en el mercado. Los hombres con $39.1 \%$ y $56.5 \%$ y las mujeres con $36.8 \%$ y $46.7 \%$ presenta la primera diferencia estadística altamente significativa entre ambos grupos

Uno de los consumos que son reflejo fuerte del estilo de personalidad y carácter es el consumo de alimentos, su correlato es el riesgo o grado de constricción de la personalidad, así ante el cuestionamiento de; Me gusta innovar en lo que consumo de alimentos o bebidas; los resultados para la muestra mexicana son $51.7 \%$ en tanto que para los participantes de Ecuador la medición arroja un valor de $62.5 \%$. En el mismo orden del trabajo los masculinos afirmaron positivamente $50.6 \%$ y $59.8 \%$, en tanto que las mujeres $52.9 \%$ y $65.2 \%$ gustan de innovar en lo que consumen en el área de alimentos.

Relacionado con este mismo rasgo y para verificar la disposición o no al riesgo se usó el reactivo Prefiero comer algo que ya conozco y he probado. Este indicador indirecto de disposición a la novedad o no. Aquí los datos altos indican no disposición a riesgo, los medios una disposición calculada de riesgo y los bajos un riesgo alto. El $71.8 \%$ de los mexicanos y $72.3 \%$ de los ecuatorianos respondieron si ante esta afirmación. 71.8\% de los mexicanos y $67.4 \%$ de los ecuatorianos respondieron de manera positiva, $71.8 \%$ de las mexicanas y $77.2 \%$ de las ecuatorianas también respondieron así.

Ambos grupos solo presentan un grado moderado de disposición al riesgo, más bien se puede afirmar un moderado sentido de riesgo.

\section{Expectativas individuales de futuro}

Se cuestionó sobre las expectativas directas de emprendimiento que se relacionan a los procesos individuales de toma de decisión, el primero Piensas que emprender un negocio es un riesgo aceptable $81 \%$ de los participantes mexicanos y $85.3 \%$ de los ecuatorianos responden que emprender un negocio es un riesgo aceptable, los hombres respondieron positivamente en el $78.7 \%$ y $84.7 \%$ de los casos, las mujeres en $83.3 \%$ y $85.9 \%$.

Ante la afirmación Vale la pena emprender con sus elementos a favor $\boldsymbol{y}$ en contra, la respuesta de los participantes mexicanos es si con $91.7 \%$ de las respuestas y $87.5 \%$ de los ecuatorianos. $89.7 \%$ de los mexicanos y $89.1 \%$ de los ecuatorianos lo afirman en tanto que $93.7 \%$ de las mexicanas y $85.9 \%$ de las ecuatorianas asumen que es un riesgo bastante aceptable desde sus expectativas.

Para finalizar el área individual se buscó detectar si visión de futuro en este tema con la afirmación Dentro de tus planes esta iniciar un negocio propio en los siguientes 5 años. 59.8 de los mexicanos y $82.1 \%$ de los participantes ecuatorianos tienen planes de emprender negocios en los siguientes 5 años, revisando por genero $58.6 \%$ de los mexicanos pretende hacerlo, $79.3 \%$ de los hombres ecuatorianos también, entre las mujeres mexicanas piensan emprender un negocio el $60.9 \%$ y entre las ecuatorianas el $84.4 \%$.

La diferencia estadística es amplia y la expectativa de las mujeres ecuatorianas de la muestra se orienta claramente hacia el emprendedurismo o emprendimiento como es denominado en la tierra del Centro del mundo.

Ante la afirmación Piensas que emprender un negocio es demasiado riesgo para una persona los respondientes mexicanos responden de manera positiva en $44 \%$, los ecuatorianos $42.9 \%$, los hombres mexicanos vs los ecuatorianos arrojan $46 \%$ vs $44.6 \%$, las mujeres 42 y $41.3 \%$ en el mismo orden de respuestas.

\section{PERSPECTIVA ENTORNO INMEDIATO}

En este apartado se incluyeron 10 aspectos que tratan sobre las influencias a favor y en contra del emprendedor y que pueden ser percibidas por los emprendedores, van desde la influencia psico social del entorno inmediato. Desde la perspectiva de influencia social se planteo

Prefiero esperar la opinión de otros sobre las cosas que deseo comprar ante esta afirmación25.3 \% de los mexicanos y 32.1de los ecuatorianos respondió afirmativamente. Por genero $25.3 \%$ de los masculinos mexicanos y $27.2 \%$ de los ecuatorianos contestaron afirmativamente. Entre las mujeres y en el mismo orden las respuestas positivas son del $23.3 \%$ y $37 \%$.

Lo anterior nos indica que para esta muestra la influencia de la opinión cercana en el consumo es baja o al menos se declara así. 
Gasto lo que puedo, después pagare. Aunque este aspecto en primera instancia es difícil de relacionar con la influencia del medio se debe atender a que el gasto y consumo están íntimamente relacionados con los hábitos familiares como elemento de interacción social. Esta parte no ha sido del todo investigada sin embargo las inferencias básicas indican que si se crece en un entorno de gasto bajo 0 alto el aprendizaje va en ese mismo sentido. Los resultados para este reactivo son $21.6 \%$ y $37.5 \%$ de mexicanos y ecuatorianos responden en forma positiva. Por genero los datos son $26.4 \%$ y $34.8 \%$ para los hombres y 16.7 y $40.2 \%$ siendo este otro de los factores de mayor diferencia estadística.

Conozco a alguien que ha iniciado un negocio en los últimos dos años. Los resultados nos indican que más de la mitad de los entrevistados en ambos países ubican en su entorno cercano a otro emprendedor $58.3 \%$ para los mexicanos y $68.5 \%$ de los ecuatorianos, por género $58 \%$ y $73.9 \%$ de los hombres ubican un emprendedor e $58.6 \%$ y $63 \%$ de las mujeres también lo hacen así.

Las habilidades percibidas están casi siempre en relación a la comparación ante otros, los respondientes contestaron a la siguiente afirmación sobre su percepción Tengo las habilidades necesarias para iniciar un negocio. 59.2\% de los mexicanos y $67.9 \%$ de los respondientes ecuatorianos responden contar con las habilidades para emprender, de ellos, los hombres en la misma secuencia del trabajo declaran en positivo el 54.6 y $66.3 \%$ de las veces, entre las mujeres la respuesta positiva fue de $63.8 \mathrm{y}$ $69.6 \%$.

Percibo buenas oportunidades de iniciar un negocio. Ante la afirmación anterior los mexicanos participantes respondieron afirmativamente en $61.5 \%$ de las veces y entre los ecuatorianos el porcentaje fue del $62 \%$ siendo esta afirmación la más cercana entre ambos grupos. Al análisis por genero los valores para los hombres $55.7 \mathrm{y}$ $64.1 \%$ y para las mujeres 67.2 y $59.8 \%$. Al igual que en la variable anterior el resultado para los masculinos mexicanos es menor al de los otros segmentos

Para emprender se requiere capacitación especializada. Ante esta afirmación $52.3 \%$ de los mexicanos y $52.7 \%$ de los ecuatorianos respondieron afirmativamente, siendo un análisis numérico y no perceptual ambos grupos afirman lo anterior solo por encima de la mitad de los valores cuartílicos, lo que da pie a un análisis más profundo en otro trabajo tratándose de una muestra de universitarios. Acaso la percepción de capacitación es desvalorada ante otros factores en emprendedurismo. Por segmento las respuestas afirmativas para hombres fueron 52.9 y $55.4 \%$ y para mujeres $51.7 \%$ y $50 \%$.
En emprendimiento siempre influye la suerte. $26.7 \%$ de los mexicanos y $40.2 \%$ de los ecuatorianos afirman la influencia de la suerte, por genero los hombres lo afirman en 32.2 y $39.1 \%$ de los respondientes, entre las mujeres 21.3 y $41.3 \%$ lo afirman así siendo esta una de las mayores diferencias entre mujeres.

Los emprendedores deben tener un respaldo económico familiar. Ante tal afirmación las respuestas para México y Ecuador son afirmativas para 60.1 y $62.5 \%$, por género y para cada grupo comparado los hombres lo afirman en 55.7 y $66.3 \%$ de los entrevistados, entre las mujeres la afirmación se dio en 64.4 y $58.7 \%$.

Un negocio debe esperar 2 a 3 años para establecerse en el mercado. Se ha medido que el fracaso de los emprendedores es la falta de un periodo medio de maduración en los negocios que se da al menos 2 años después de iniciado. Los datos de respuesta de los grupos participantes informan lo siguiente para la muestra mexicana la afirmación positiva es $52.3 \%$ y para la parte ecuatoriana es del $64.1 \%$, por genero los mexicanos y ecuatorianos contestaron afirmativamente en 54.6 y $62 \%$ de los cados, entre las mujeres y en el mismo orden $50 \mathrm{y}$ $66.3 \%$ respectivamente.

Finalmente, en esta sección de influencias cercanas que facilitan o limitan el emprendimiento se evalúa la afirmación sobre la percepción del medio cercano.

Mi entorno facilita el emprendimiento. Las respuestas generales son: para México $50.3 \%$ afirman que el entorno cercano facilita el emprendimiento, para los ecuatorianos la respuesta positiva fue del $52.7 \%$. Entre hombres 43.7 y $55.4 \%$, entre las mujeres 56.9 y $50 \%$. Lo anterior nos indica que solo la mitad de los entrevistados en ambas muestras detecta un ambiente cercano positivo para realizar una actividad emprendedora.

\section{NEGOCIOS POR EMPRENDER Y MOTIVACIONES PARA ELLO}

Se realizó un cruce que incluye solo a aquellos que estiman emprenderán un negocio en los siguientes 5 años, de la muestra total de 532 respondientes 359 estiman emprenderán un negocio en el periodo mencionado lo que significa el $67.5 \%$ del total de participantes. Para efectuar un análisis comparativo por país las muestras se tomaron como $100 \%$ separando Ecuador y México con los resultados referidos a continuación.

El $50.5 \%$ de los participantes Mexicanos y el $82 \%$ de los Ecuatorianos pretenden establecer un negocio en los siguientes 5 años. Por genero los datos para hombres mexicanos y ecuatorianos respectivamente son $58.6 \%$ y 
ISSN: 1794-9920 Julio - Diciembre de 2018

Volumen 18 Número 2, Año 2018 Págs. 39 - 49

$79.3 \%$, entre las mujeres del total de respondientes el $58 \%$ de las mexicanas y el $84.8 \%$ de las ecuatorianas participantes emprenderán un negocio de acuerdo con sus declaraciones. Los negocios que pretenden emprender por país se representan en la gráfica 1.

Gráfica 1. Negocio que emprenderán los entrevistados mexicanos

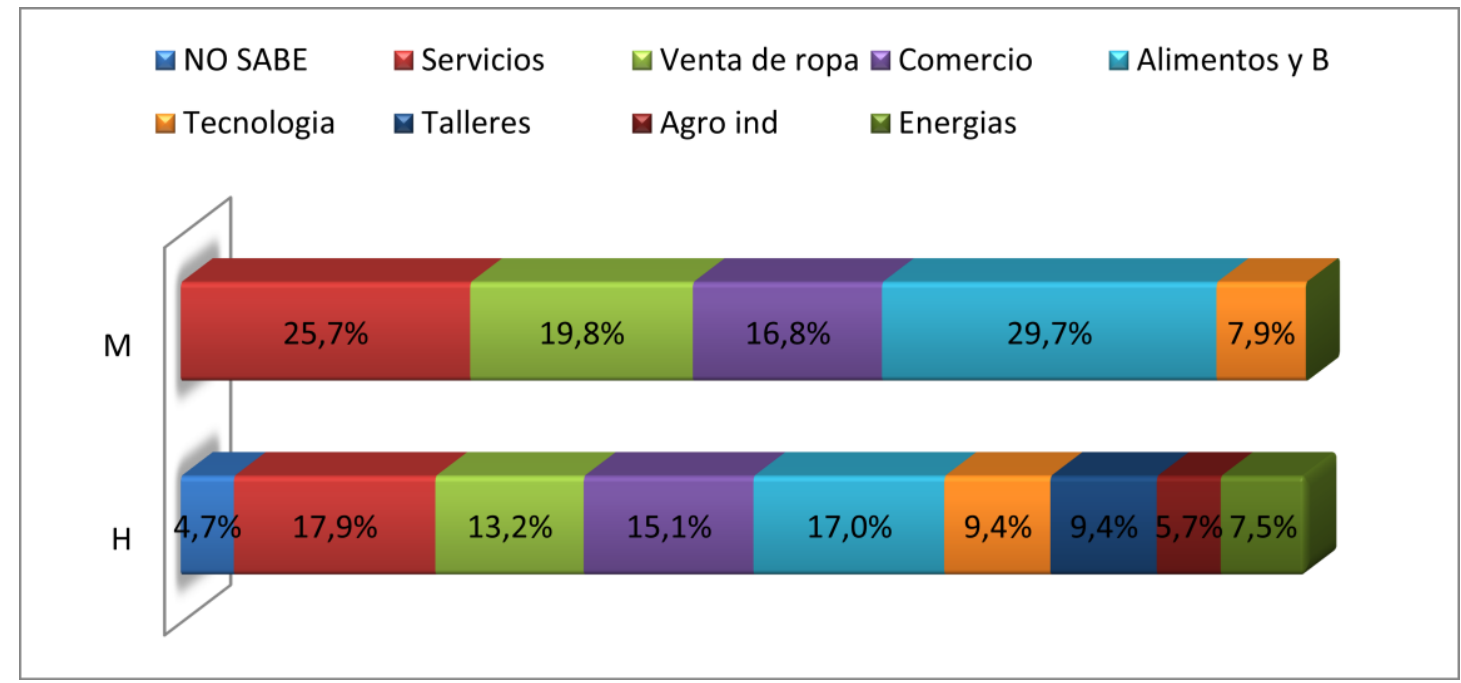

Fuente: elaboración propia

La gráfica muestra los negocios a establecer, se totalizo el $100 \%$ de los que respondieron pretenden establecer negocios en los siguientes 5 años.

Las mujeres mexicanas estiman establecer un negocio de alimentos y bebidas con el $29.7 \%$ de los negocios a establecer, en segundo lugar, estiman iniciar una empresa de servicios (desde servicios de diseño hasta jurídicos, la gama contempla otros servicios), por último y con $19.8 \%$ las mujeres mexicanas se enfocan en un negocio de venta de ropa.

Los hombres mexicanos establecen con 17.9\% como primera opción de negocio los servicios, en segundo lugar, con $17 \%$ establecimientos de alimentos y bebidas y en tercer lugar el comercio en diversas modalidades. Otros negocios se aprecian con menores porcentajes. En el caso de los participantes ecuatorianos los negocios que planean establecer se presentan en la gráfica 2.

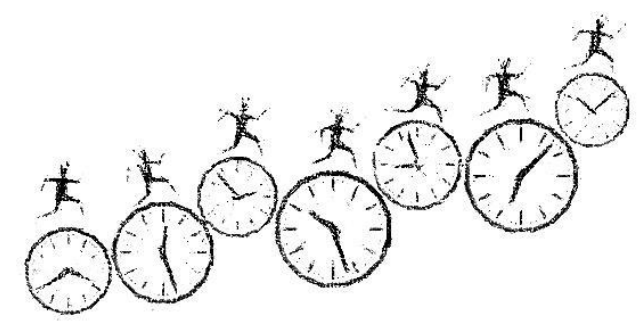


Gráfica 2. Negocios que emprenderán los entrevistados ecuatorianos

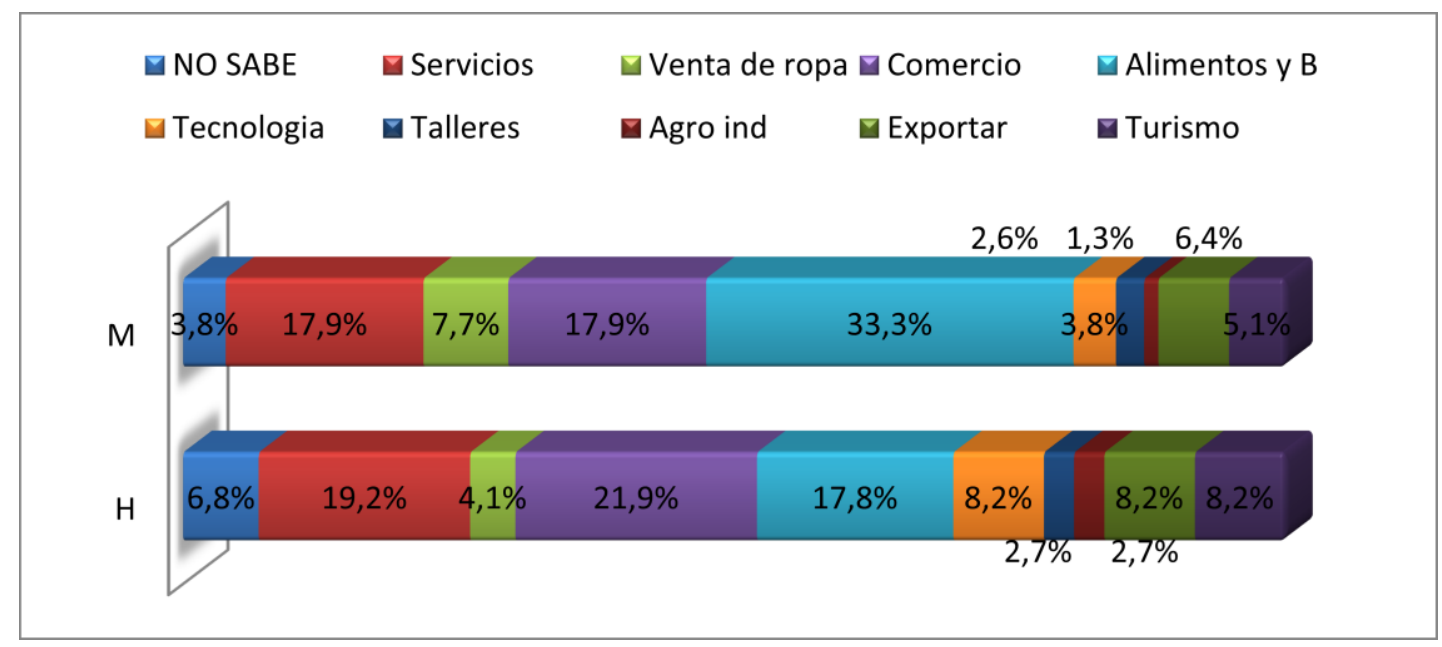

Fuente: elaboración propia

La gráfica muestra los negocios a establecer, se totalizo el $100 \%$ de los que respondieron pretenden establecer negocios en los siguientes 5 años.

Las mujeres ecuatorianas en un $33.3 \%$ estiman establecer en los siguientes 5 años un negocio en el ramo de alimentos y bebidas, seguido de comercios o servicios con el $17.9 \%$ de las participantes. Los hombres de esta nacionalidad estiman establecer Un comercio como primera opción con el $21.9 \%$ de las respuestas, seguido de un establecimiento de servicios con el $19.2 \%$, en tercer lugar, estiman establecer negocios de alimentos y bebidas en el $17.8 \%$ de los casos. Otros establecimientos emprendedores se aprecian con menores porcentajes.

\section{CAPACITACIÓN PARA PODER EMPRENDER}

Ante los cuestionamientos sobre las áreas de capacitación específica que requerían para emprender ese negocio planeado el top of mind de capacitación solicitada es para los diferentes países y por género. Los hombres mexicanos requieren capacitación en administración $16.7 \%$, economía y finanzas $14.7 \%$, tecnologías $13.7 \%$, mercadotecnia $9.8 \%$, no saben que capacitación $9.8 \%$ y el restante aparece con menores valores para diversas capacitaciones.

Las mujeres mexicanas declaran necesidad de capacitación en administración 22.8\%, 13.9\% en economía y finanzas, $10.9 \%$ en diseño y moda, $9.9 \%$ en gastronomía, $9.9 \%$ no sabe que capacitación requerirá y las restantes en diversas áreas y con valores menores hasta completar el $100 \%$. aparece ser su jefe con el $11.8 \%$, en cuarto lugar gusto por emprender con $10.8 \%$ y en quinto lugar la familia con $10.8 \%$. El mayor motivador para las mujeres mexicanas es el dinero con el $18.8 \%$ de las afirmaciones, en segundo lugar con $15.8 \%$ de las menciones esta la familia, en tercer lugar y con $10.9 \%$ aparece el reconocimiento y poder, otras motivaciones aparecen con $9 \%$ de menciones o menos.

En el caso de Ecuador el principal motivador con un $24.7 \%$ se ubica la familia, enseguida $20 \%$ declaran como motivador la superación personal, en tercero con $11 \%$ de las menciones está el dinero, con $9.6 \%$ ser su jefe. Las otras menciones tienen proporciones menores en las menciones.

Las mujeres ecuatorianas declaran que la superación es su principal motivador $21.8 \%$ afirman de tal manera, el segundo motivador es con $14.1 \%$ la familia, con $11.5 \%$ el gusto por emprender, con $9 \%$ de las declaraciones esta ser su jefe y con $7.7 \%$ el dinero como motivador. Otras motivaciones aparecen con menores porcentajes.

\section{HALLAZGOS}

$\checkmark \quad$ Existe una diferencia estadística de más de 13 puntos en el aspecto de intentar comprar lo más nuevo en el mercado, presentándose valores mayores entre los ecuatorianos.

$\checkmark \quad$ Más del $80 \%$ de los entrevistados aceptan que el riesgo de emprender un negocio es aceptable para ellos. 
$\checkmark$ Casi 6 de cada 10 entrevistados universitarios mexicanos estiman emprender un negocio en los siguientes 5 años, en tanto que más de 8 de cada 10 entrevistados ecuatorianos intentara emprender un negocio en los siguientes 5 años.

$\checkmark$ El $84.8 \%$ de las mujeres universitarias ecuatorianas de la muestra intentaran emprender un negocio en los siguientes 5 años.

$\checkmark \quad$ Las expectativas individuales dan pie a pensar en una buena posibilidad de emprendimiento en el área de evaluación individual y personal.

$\checkmark$ Se detectó que los participantes ecuatorianos están más dispuestos al riesgo de deuda, por genero los datos son $26.4 \%$ y $34.8 \%$ para los hombres y $16.7 \%$ y $40.2 \%$ para las mujeres siendo este otro de los factores de mayor diferencia estadística

$\checkmark \quad$ El nivel más bajo en declaración de habilidad para emprender un negocio se encuentra en hombres mexicanos con el $54.6 \%$ y casi 10 puntos de diferencia por debajo de los otros grupos participantes.

$\checkmark$ Percibir buenas oportunidades para iniciar un negocio es una de las dos variables más cercanas a total al comparar los grupos con valores de $61.5 \%$ y $62 \%$ mexicanos y ecuatorianos en la muestra de universitarios estudiada.

$\checkmark \quad$ La variable más cercana entre los grupos es para emprender un negocio se requiere capacitación especializada con valores de 52.3 y $52.7 \%$ en el mismo orden. Cabe resaltar aquí que esta necesidad detectada (la capacitación especializada) es apenas mayor a la mitad de los respondientes para ambos países.

$\checkmark$ Seis de cada diez universitarios afirman que se debe tener un respaldo económico familiar para un negocio emprendedor.

$\checkmark$ Uno de cada dos entrevistados percibe un ambiente cercano que favorece el emprendedurismo.

$\checkmark \quad 67.5 \%$ de los jóvenes entrevistados pretenden emprender un negocio en los siguientes 5 años.

$\checkmark$ Servicios, alimentos y comercio general son los negocios que emprenderán los mexicanos y ecuatorianos

$\checkmark$ Economía y finanzas, administración, mercadotecnia y relaciones públicas son las capacitaciones más requeridas pop los futuros emprendedores

$\checkmark \quad$ La capacitación en idiomas aun no es detectada como urgente, así como la de tecnologías.
La principal motivación es la superación personal con un promedio de $17.2 \%$, en segundo lugar, motivacional se encuentra la familia con $16.1 \%$, en tercero el dinero con el $13.3 \%$ y en cuarto lugar de motivaciones emprendedoras con $9.9 \%$ se encuentra el gusto por emprender.

\section{CONCLUSIONES:}

Son múltiples los factores que pueden motivar el emprendimiento, entre ellos los rasgos de personalidad como la superación personal ante la situación social hoy es el elemento de mayor declaratoria por parte de aquellos que pretenden emprender en ambas muestras de estudiantes participantes en esta investigación.

El segundo factor motivacional lo constituye la familia como elemento impulsor de la mejora y la toma de riesgo para emprender.

Para ambos grupos participantes, el dinero es el tercer factor que impulsa al riesgo y la intensión de crear un negocio en los siguientes cinco años. Ante la falta de estabilidad y la competencia por los puestos de trabajo el gusto por emprender y ser su jefe, son factores que también impulsaran a los futuros emprendedores.

En cuanto a las capacitaciones que los participantes detectan como necesarias para emprender se encuentran las de administración, finanzas, mercadotecnia y relaciones públicas, más que aquellas muy especializadas no importando el emprendimiento que se pretenda.

Los futuros emprendedores declaran la intensión de desarrollarse en áreas de gastronomía, servicios y comercio en general más que aquellas que requieren gran especialización específica. Se detecta que los participantes ecuatorianos presentan una mayor disposición al emprendimiento que la muestra de participantes mexicanos. Seis de cada diez participantes mexicanos pretenden emprender un negocio en los siguientes cinco años, en tanto que ocho de cada diez ecuatorianos pretenden hacerlo así.

Para futuras investigaciones se recomienda un análisis de los factores culturales y de genero pues las declaraciones femeninas se encaminan a actividades asociadas con el rol femenino en la dinámica tradicional de las sociedades y las declaraciones masculinas se alejan de estos conceptos para tratar de incursionar en emprendedurismo en áreas asociadas con el concepto tradicional de machismo. 
Finalmente, el concepto de emprendedurismo se ha insertado en estos grupos como una opción viable para lograr la superación en la sociedad, no se percibe como moda, ni se cuestiona al menos por estas sociedades. El segundo factor de importancia en la intensión de ser emprendedor es el apoyo a la familia y el mejoramiento en la escala social, finalmente el dinero se constituye como otro de los impulsores a la intensión de emprender.

\section{REFERENCIAS:}

Amorós (2007). Comportamiento organizacional, En busca del desarrollo de ventajas competitivas, Universidad Católica San Toribio de Mogrovejo. USAT. Chyclayo. Perú.

Cantillo R. (1755) / (2011). Essai sur la nature du commerce en général. Institut Coppet. Disponible en: https://www.institutcoppet.org/wpcontent/uploads/2011/12/Essai-sur-la-nature-ducommerce-en-gener-Richard-Cantillon.pdf.

Cordero J. Astudillo,S. Carpio,X. Delgado,J. Amon, O. (2011). Analisis de los factores que influyen en el emprendimiento y sostenibilidad de las empresas del área urbana de la ciudad de Cuenca, Ecuador. MASKANA. Vol2, $n^{\circ} 2$. Disponible en: http://dspace.ucuenca.edu.ec/bitstream/123456789/5 412/1/MASKANA\%20si7285\%20\%283\%29.pdf

Espíritu, R. Moreno, H. (2014) La influencia de los rasgos de personalidad, control interno y necesidad de logro en la intención emprendedora: Un estudio empírico con estudiantes universitarios. XV Congreso internacional de contaduría, administración e informática. ANFECA. Disponible en: http://congreso.investiga.fca.unam.mx/docs/xv/do cs/7.pdf

Hernández. Fernández. Baptista (2010). Metodología de la investigación. Quinta edición. Mc Graw Hill. Mex.

Huerta (1986). El empresario. Kirzner. Lecturas de Economía Política, vol. I, Unión Editorial, Madrid http://www.eumed.net/cursecon/textos/Kirzner_e mpresario.pdf 150918
Junta de Andalucía (2015). Libro blanco del emprendimiento. I Foro Internacional de Emprendimiento Andalucía Emprende. Disponible en: https://www.andaluciaemprende.es/wpcontent/uploads/2015/02/LIBRO-BLANCOEMPRENDIMIENTO-ES.pdf

Kotler \& Armstrong (2008). Fundamentos de Marketing. Printice Hall. Octava Edición. México

Parra, Rubio y López (2017). Factores distintivos del emprendimiento que propiciaron el éxito: casos de estudio en empresarios de Ibagué, Tolima. Pensamiento y gestión No 43. Disponible en: http://rcientificas.uninorte.edu.co/index.php/pensa miento/article/view/9216

Rubio, J. (2013). Factores clave de éxito asociados al emprendimiento de base tecnológica en la ciudad de Manizales. Un análisis de la cadena de emprendimiento. Universidad nacional de Colombia. Disponible en: http://www.bdigital.unal.edu.co/11859/1/7709522. 2013.pdf

Suarez, J., Pedroza , I. (2016). Evaluación de la personalidad emprendedora: Situación actual y líneas de futuro. Papeles del psicólogo vol $37 n^{\circ}$ 1. Madrid España. Disponible en: http://www.redalyc.org/pdf/778/77844204008.pdf

Torralba L. (2014) Factores clave en el desarrollo del emprendimiento. Facultad de ciencias económicas y empresariales. Universidad pontificia Comillas Madrid. Disponible: https://repositorio.comillas.edu/xmlui/bitstream/ha ndle/11531/61/TFG000007.pdf?sequence=1 050918 